\title{
Biography: Professor Mehran Anvari, 00nt, MB BS, PhD, FRCSC, FACS
}

\author{
Mehran Anvari ${ }^{1}$ (D)
}

Published online: 3 December 2019

(C) Springer Science+Business Media, LLC, part of Springer Nature 2019

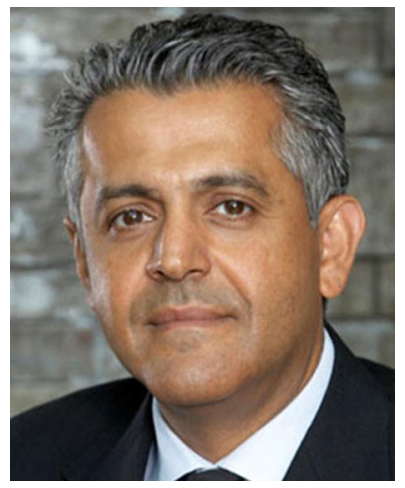

Dr. Anvari obtained his medical degree from University of Newcastle upon Tyne, UK, and completed his surgical training at McMaster University, Canada, in 1989. He then completed and obtained a $\mathrm{PhD}$ degree from University of Adelaide in Australia. His thesis was on neuroendocrine control of gastric emptying and effect of gastric surgery. He joined McMaster University as an Assistant Professor in 1992 and due to his accomplishments was promoted to the rank of Associate Professor in 1996 and became a Full Professor with tenure in 2000.

In 2004, he was appointed to the newly created Chair in Minimally Invasive Surgery and Surgical Innovation, and in 2005, he became the Founding Director of the McMaster Institute for Surgical Invention, Innovation and Education. $\mathrm{He}$ is also the Founding Director of the Centre for Minimal Access Surgery (CMAS) and the Scientific Director and CEO of the Centre for Surgical Invention and Innovation (CSii). In 2016, CSii partnered with MDA to create Insight Medbotics Canada Corporation (IMCC) - a start-up company to commercialize a new generation of intelligent robotic systems.

Mehran Anvari

anvari@mcmaster.ca

1 McMaster University, Hamilton, Ontario, Canada
In mid-2000, Dr. Anvari advocated for increased federal and provincial funding for bariatric services and in 2009 was successful in securing significant programmatic funding for bariatric services in Ontario. He was appointed as Provincial Clinical Lead and Chair of the Ontario Bariatric Network and helped establish 10 Bariatric Centers of Excellence across Ontario. As one of the founding members of the Canadian Association of Bariatric Surgeons and Physicians, Dr. Anvari has been instrumental in expanding bariatric services in Canada. From 2012 to 2018, he served as a member and the treasurer of the North American Chapter of IFSO and also served on the Board of IFSO from 2015 to 2018. Dr. Anvari is an active member of CABPS, ASMBS, and IFSO.

Dr. Anvari's other major interest has been expanding the use of minimally invasive surgeries and robotic surgery. His body of work has been internationally recognized and acknowledged. Time Magazine has touted CMAS as "sculpting the next frontier of medicine." Through CMAS, the first of its kind in Canada, Dr. Anvari has promoted the use of minimal access techniques in all surgical specialties and has focused on improving patient outcomes, minimizing the physical, emotional, and financial impact of surgical procedures on patients, and reducing hospital admissions and the associated costs. His dedication to the ongoing training and mentoring of surgeons through CMAS has resulted in the creation of a series of continuing education programs that are unrivaled in scope, subject matter, and participation. Under his direction, CMAS has trained over 1200 surgeons, providing them with their primary source of training in the newest techniques and applications in the use of minimal access surgery.

Professor Anvari was one of the first surgeons in Canada to use robotics in surgery (1996), and in 2003, he established the world's first telerobotic surgical service linking St. Joseph's Healthcare Hamilton and a community hospital, North Bay General. This work received international and national news coverage and even today is quoted and described in the 8th grade science textbook used by Ontario students. He was the Chief Scientific Officer for the NEEMO 7 (2004) and NEEMO 9 (2006) missions. These missions were joint 
projects of McMaster, the Canadian Space Agency, and NASA that were tasked with testing the ability of new robotic and telesurgical technology to allow a nonphysician to perform assisted surgery in a contained environment that simulates conditions in space. He is recognized as one of the pioneers of telerobotic surgery and is the Past President of MIRA, the association representing robotic surgeons worldwide. Prof. Anvari is the Editor in Chief of the International Journal of Medical Robotics and Computer Assisted Surgery (Wiley publication), the only indexed robotic journal in the world.

Professor Anvari was awarded an Honorary Doctorate from University of Toledo in 2017 and was inducted into the Royal College of Physicians and Surgeons of Glasgow in 2010 and the Israeli College of Surgeons in 2017. He was the recipient of the 2009-2010 ORION Award for Leadership, the Government of Ontario Diamond Award for
Innovation in Technology, the Government of Canada Gold Medal of Distinction for Telerobotic Surgery, the McMaster Innovator of the Year Award (2009), and the NASAInternational Space Station (ISS) Research and Development Award for Biology and Medicine for the development of IGAR in July 2015.

Dr. Anvari lives in Hamilton, Ontario, with his wife Sima and his 4 children Sama, Bijan, Naseem, and Nava. He is very engaged in a number of community initiatives and served on the Board of Hillfield Strathallan College. Dr. Anvari organizes an open annual innovation conference for high school and university students to encourage interest in STEM subjects and innovation. He is currently a member of the National Body of the Baha'is of Canada.

In June of 2017, Lt. Gov. of Ontario Elizabeth Dowdeswell appointed Professor Anvari to the Order of Ontario, the highest civilian honor bestowed in Ontario, Canada. 